\title{
ÜBER DIE LAGE DER NULLSTELLEN EINES ABSTANDS- POLYNOMS UND SEINER DERIVIERTEN
}

GYULA V. SZ. NAGY

1. Der Begriff eines Abstandspolynoms. Ein Abstandspolynom $F(x, y, z)$ n-ten Grades in dem rechtwinkligen Koordinatensystem $x, y, z$ ist ein reelles Polynom der drei Veränderlichen von der Form

$$
\begin{aligned}
& F(x, y, z)=C \prod_{k=1}^{n} d_{k}(x, y, z), \\
& d_{k}(x, y, z)=\left(x-x_{k}\right)^{2}+\left(y-y_{k}\right)^{2}+\left(z-z_{k}\right)^{2}, \quad C>0 .
\end{aligned}
$$

Das Abstandspolynom $F(x, y, z)$ ist also eine nichtnegative reelle Funktion von der Form

$$
F(x, y, z)=C\left(x^{2 n}+y^{2 n}+z^{2 n}\right)+\Phi(x, y, z),
$$

wo $\Phi$ ein reelles Polynom ist, das für die drei Veränderlichen bzw. für die einzelnen Veränderlichen höchstens den Grad $2 n-1$ bzw. $2 n-2$ besitzt.

Das Abstandspolynom $F(z, y, z)$ besitzt $n$ Nullstellen, und zwar die Punkte

$$
Q_{k}=\left(x_{k}, y_{k}, z_{k}\right) \quad(k=1,2, \cdots, n) .
$$

Ausser den Nullstellen nimmt das Abstandspolynom $F(x, y, z)$ in jedem (reellen) Punkte des Raumes einen positiven Wert an.

Das Polynom $d_{k}(x, y, z)=\left(x-x_{k}\right)^{2}+\left(y-y_{k}\right)^{2}+\left(z-z_{k}\right)^{2}$ ist ein Abstandspolynom ersten Grades und ist ein Wurzelfaktor des Abstandspolynoms $F(x, y, z) n$-ten Grades. $F$ ist also ein Produkt von $n$ Wurzelfaktoren. Kommt der Wurzelfaktor $d_{k}$ unter den $n$ Wurzelfaktoren $p$-mal vor $(p \geqq 1)$, so ist $Q_{k}$ eine $p$-fache Nullstelle von $F$. Dann ist

$$
F(x, y, z)=d_{k}^{p}(x, y, z) \cdot G(x, y, z),
$$

wo $G(x, y, z)$ ein Abstandspolynom $(n-p)$-ten Grades ist, für welches die Ungleichung $G\left(x_{k}, y_{k}, z_{k}\right) \neq 0$ besteht.

Jedes Abstandspolynom $f(x, y, z)=d_{k}(x, y, z)$ ersten Grades genügt offenbar der Identät

$$
f_{x}^{2}+f_{y}^{2}+f_{z}^{2}=4 \cdot f .
$$

Hat eine ganze rationale Funktion $2 n$-ten Grades von der Form (2)

Received by the editors March 1, 1948. 
einen Faktor zweiten Grades, so hat dieser Faktor leicht ersichtlich die Form $f(x, y, z)=c\left(x^{2}+y^{2}+z^{2}+a_{1} x+a_{2} y+a_{3} z+a_{4}\right), c \neq 0$. Das Abstandspolynom $F(x, y, z) n$-ten Grades ist eine Verallgemeinerung eines Polynoms $f(z)$ der komplexen Veränderlichen $z$.

Sind nämlich

$$
\begin{aligned}
f(z) & =\left(z-z_{1}\right)\left(z-z_{2}\right) \cdots\left(z-z_{n}\right), \\
z & =x+i y, z_{k}=x_{k}+i y_{k} \quad(k=1,2, \cdots, n), \\
f(x+i y) & =U(x, y)+i V(x, y), \\
\bar{f}(x-i y) & =U(x, y)-i V(x, y),
\end{aligned}
$$

so ist

$$
\begin{aligned}
F(x, y) & =U^{2}(x, y)+V^{2}(x, y)=|f(z)|^{2} \\
& =\prod_{k=1}^{n}\left|z-z_{k}\right|^{2}=\Pi\left[\left(x-x_{k}\right)^{2}+\left(y-y_{k}\right)^{2}\right]
\end{aligned}
$$

ein Abstandspolynom $n$-ten Grades in der $x, y$ Koordinatenebene.

Ein Abstandspolynom $F(x, y, z)$ geht durch eine orthogonale Transformation der Veränderlichen $x, y, z$ offenbar wieder in ein Abstandspolynom über. Daraus folgt, dass ein Abstandspolynom, dessen Nullstellen in einer Ebene liegen, durch eine lineare Transformation sich auf die Form

$$
F(x, y, z)=C \prod_{k=1}^{n}\left[\left(x-x_{k}\right)^{2}+\left(y-y_{k}\right)^{2}+z^{2}\right]
$$

bringen lässt.

2. Die Derivierte eines Abstandspolynoms. Ist $F(x, y, z)$ ein Abstandspolynom $n$-ten Grades, so ist das Polynom (4n-2)-ten Grades

$$
H(x, y, z)=F_{x}^{2}(x, y, z)+F_{y}^{2}(x, y, z)+F_{z}^{2}(x, y, z)
$$

durch $4 \cdot F(x, y, z)$ teilbar, sodass die Funktion

$$
\begin{aligned}
F^{\prime}(x, y, z) & =\frac{H(x, y, z)}{4 F(x, y, z)} \\
& =\frac{F_{x}^{2}(x, y, z)+F_{y}^{2}(x, y, z)+F_{z}^{2}(x, y, z)}{4 \cdot F(x, y, z)}
\end{aligned}
$$

ein Polynom 2(n-1)-ten Grades von den Veränderlichen $x, y, z$ ist. 
Diese Funktion $F^{\prime}(x, y, z)$ wird als Derivierte des Abstandspolynoms $F(x, y, z)$ genannt.

Ist

$$
\begin{aligned}
F(x, y, z) & =f^{p}(x, y, z) \cdot G(x, y, z), \\
f(x, y, z) & =d_{k}(x, y, z), \quad p \geqq 1, \quad G\left(x_{k}, y_{k}, z_{k}\right) \neq 0
\end{aligned}
$$

so ist

$$
\begin{aligned}
H(x, y, z)= & F_{x}^{2}+F_{y}^{2}+F_{z}^{2}=p^{2} f^{2 p-2} G^{2}\left(f_{x}^{2}+f_{y}^{2}+f_{z}^{2}\right) \\
& +2 p f^{2 p-1}\left(f_{x} G_{x}+f_{y} G_{y}+f_{z} G_{z}\right)+f^{2 p}\left(G_{x}^{2}+G_{y}^{2}+G_{z}^{2}\right) \\
= & f^{2 p-1}\left[4 p^{2} G^{2}+2 p G\left(f_{x} G_{x}+f_{y} G_{y}+f_{z} G_{z}\right)\right. \\
& \left.+f\left(G_{x}^{2}+G_{y}^{2}+G_{z}^{2}\right)\right] .
\end{aligned}
$$

Daraus folgt, dass $H$ durch die $(2 p-1)$-te Potenz des Wurzelfaktors $f=d_{k}$ teilbar ist, durch eine höhere Potenz von $f$ aber nicht, weil die $z$ wischen den eckigen Klammerzeichen stehende Funktion im Punkte $Q_{k}=\left(x_{k}, y_{k}, z_{k}\right)$ den Wert $4 p^{2} G^{2}\left(x_{k}, y_{k}, z_{k}\right) \neq 0$ annimmt.

Hieraus folgt, dass $H$ durch jeden Wurzelfaktor von $F$ und deshalb auch durch $F$ teilbar ist und dass eine $p$-fache Nullstelle $Q_{k}$ des Abstandspolynoms $F$ eine $2 p-1-p=(p-1)$-fache Nullstelle der Derivierten ist, weil $F^{\prime}$ durch die $(p-1)$-te Potenz von $d_{k}$ teilbar ist.

Nach der Regel der logarithmischen Differentiation ist

$$
\frac{F_{x}}{F}=\frac{\partial}{\partial x} \log F=\frac{\partial}{\partial x} \sum_{k=1}^{n} \log d_{k}=\sum_{k=1}^{n} \frac{1}{d_{k}} \cdot \frac{\partial d_{k}}{\partial x}=2 \cdot \sum_{k=1}^{n} \frac{x-x_{k}}{d_{k}} .
$$

Es gelten also für die Nullstellen $(x, y, z)$ der Derivierten $F^{\prime}(x, y, z)$, die von den Nullstellen des Abstandspolynoms $F(x, y, z)$ abweichen, die Gleichungen

$$
\begin{gathered}
\frac{F_{x}}{2 \cdot F}=\sum_{k=1}^{n} \frac{x-x_{k}}{d_{k}}=0, \quad \frac{F_{y}}{2 \cdot F}=\sum_{k=1}^{n} \frac{y-y_{k}}{d_{k}}=0, \\
\frac{F_{z}}{2 \cdot F}=\sum_{k=1}^{n} \frac{z-z_{k}}{d_{k}}=0 .
\end{gathered}
$$

Auf Grund der Bezeichnungen (6) und (7) ist

$$
F^{\prime}(x, y)=\frac{F_{x}^{2}(x, y)+F_{y}^{2}(x, y)}{4 \cdot F(x, y)}=\left|f^{\prime}(z)\right|^{2},
$$

weil

$$
F_{x}=2\left(U U_{x}+V V_{x}\right), \quad F_{y}=2\left(U U_{y}+V V_{y}\right),
$$




$$
f^{\prime}(z)=U_{x}+i V_{x}=V_{y}-i U_{y}, \quad U_{x}=V_{y}, \quad U_{y}=-V_{x}
$$

und deshalb

$$
\begin{aligned}
F_{x}^{2}+F_{y}^{2} & =4\left[\left(U U_{x}+V V_{x}\right)^{2}+\left(U U_{y}+V V_{y}\right)^{2}\right] \\
& =4\left(U^{2}+V^{2}\right)\left(U_{x}^{2}+V_{x}^{2}\right)=4 \cdot F(x, y) \cdot F^{\prime}(x, y)
\end{aligned}
$$

sind.

Die Derivierte des Abstandspolynoms $F(x, y)$ ist also wieder ein Abstandspolynom der Veränderlichen $x, y$, falls $n>1$ ist.

Sind $z_{k}=0(k=1,2, \cdots, n)$, so erhält man für die von den Nullstellen des Abstandspolynoms $F(x, y, z)$ abweichenden Nullstellen der Derivierten $F^{\prime}(x, y, z)$ aus (10) die Gleichungen

$$
\begin{aligned}
z=0, & \sum_{k=1}^{n} \frac{x-x_{k}}{\left(x-x_{k}\right)^{2}+\left(y-y_{k}\right)^{2}}=0, \\
& \sum_{k=1}^{n} \frac{y-y_{k}}{\left(x-x_{k}\right)^{2}+\left(y-y_{k}\right)^{2}}=0 .
\end{aligned}
$$

Die Nullstellen der Derivierten $F^{\prime}(x, y, z)$ stimmen also im Falle $z_{k}=0(k=1,2, \cdots, n)$ mit den Nullstellen der Funktion $F^{\prime}(x, y)$ von (11) überein. Ist

$$
\begin{gathered}
f^{\prime}(z)=n\left(z-z_{1}^{\prime}\right)\left(z-z_{2}^{\prime}\right) \cdots\left(z-z_{n-1}^{\prime}\right) \\
z=x+i y, \quad z_{h}^{\prime}=x_{h}^{\prime}+i y_{h}^{\prime} \quad(h=1,2, \cdots, n-1)
\end{gathered}
$$

so ist

$$
F^{\prime}(x, y)=n^{2} \prod_{h=1}^{n-1}\left[\left(x-x_{h}^{\prime}\right)^{2}+\left(y-y_{h}^{\prime}\right)^{2}\right]=F_{1}(x, y, 0)
$$

und deshalb ist auch die Derivierte

$$
F^{\prime}(x, y, z)=n^{2} \prod_{h=1}^{n-1}\left[\left(x-x_{h}^{\prime}\right)^{2}+\left(y-y_{h}^{\prime}\right)^{2}+z^{2}\right]=F_{1}(x, y, z)
$$

ein Abstandspolynom.

Naheliegend ist die Vermutung, das die Derivierte jedes Abstandspolynoms $F(x, y, z) n$-ten Grades $(n \geqq 2)$ stets ein Abstandspolynom ist.

\section{Der Gauss-Lucas-sche Satz über Abstandspolynome.}

I. Die (reellen) Nullstellen der Derivierten eines Abstandspolynoms $F(x, y, z)$ fallen in die konvexe Hülle der Nullstellen von $F(x, y, z)$. Die Randfä̈che dieser konvexen Hülle kann nur dann eine Nullstelle 
$P_{0}$ der Derivierten enthalten, wenn $P_{0}$ mit einer Nullstelle von $F(x, y, z)$ zusammenfällt.

Bedeutet nämlich $P_{0}=\left(x_{0}, y_{0}, z_{0}\right)$ eine Nullstelle der Derivierten des Abstandspolynoms $F(x, y, z)$, für welche $F\left(x_{0}, y_{0}, z_{0}\right) \neq 0$ gilt und sind

$$
m_{k}=\frac{1}{\left(x_{0}-x_{k}\right)^{2}+\left(y_{0}-y_{k}\right)^{2}+\left(z_{0}-z_{k}\right)^{2}}, \quad M=\sum_{k=1}^{n} m_{k},
$$

so lassen sich die Gleichungen (10) in der Form

$$
\sum_{k=1}^{n} m_{k}\left(x_{0}-x_{k}\right)=0, \quad \sum_{k=1}^{n} m_{k}\left(y_{0}-y_{k}\right)=0, \quad \sum_{k=1}^{n} m_{k}\left(z_{0}-z_{k}\right)=0
$$

oder

$$
M x_{0}=\sum_{k=1}^{n} m_{k} x_{k}, \quad M y_{0}=\sum_{k=1}^{n} m_{k} y_{k}, \quad M z_{0}=\sum_{k=1}^{n} m_{k} z_{k}
$$

schreiben.

Daraus folgt der Satz, weil $P_{0}$ der Schwerpunkt der Punkte $Q_{k}$ von den Massen $m_{k}(k=1,2, \cdots, n)$ ist. I.

Aus den Gleichungen (12) folgt auch die folgende Form des Satzes

II. Jede Nullstelle $P_{0}=\left(x_{0}, y_{0}, z_{0}\right)$ der Derivierten des Abstandspolynoms $F(x, y, z)$ für welche $F\left(x_{0}, y_{0}, z_{0}\right) \neq 0$ gilt, ist eine Gleichgewichtslage eines materiellen Punktes $P$ des Raumes, auf welchen die Nullstellen $Q_{k}$ von $F(x, y, z)$ dem Abstand umgekehrt proportionale abstossende Kräfte ausüben.

Bei geeignet gewähltem Masssystem sind nämlich die linken Seiten der Gleichungen (12) den Komponenten der Resultante dieser Kräfte in bezug auf den Punkt $P_{0}$ gleich.

Bezeichnet $Q_{k}^{\prime}=\left(x_{k}^{\prime}, y_{k}^{\prime}, z_{k}^{\prime}\right)$ den Spiegelpunkt von $Q_{k}=\left(x_{k}, y_{k}, z_{k}\right)$ in bezug auf die Kugel vom Mittelpunkt $P_{0}$ und vom Einheitsradius, so sind

$$
\begin{gathered}
x_{k}^{\prime}-x_{0}=m_{k}\left(x_{k}-x_{0}\right), \quad y_{k}^{\prime}-y_{0}=m_{k}\left(y_{k}-y_{0}\right), \\
z_{k}^{\prime}-z_{0}=m_{k}\left(z_{k}-z_{0}\right) .
\end{gathered}
$$

Für diese Punkte $Q_{k}^{\prime}$ bestehen also die Gleichungen

$$
\begin{array}{rlrl}
\sum\left(x_{k}^{\prime}-x_{0}\right) & =0, \quad \sum\left(y_{k}^{\prime}-y_{0}\right)=0, & \sum\left(z_{k}^{\prime}-z_{0}\right)=0, \\
\text { oder } n x_{0}=\sum x_{k}^{\prime}, \quad n y_{0}=\sum y_{k}^{\prime}, & n z_{0}=\sum z_{k}^{\prime} .
\end{array}
$$


Daraus folgt der Satz

III. Ist $P_{0}=\left(x_{0}, y_{0}, z_{0}\right)$ eine (reelle) Nullstelle der Derivierten des Abstandspolynoms $F(x, y, z)$ n-ten Grades mit den Nullstellen $Q_{1}$, $Q_{2}, \cdots, Q_{n}$, für welche $F\left(x_{0}, y_{0}, z_{0}\right) \neq 0$ gilt, und bezeichnet $Q_{k}^{\prime}$ den Spiegelpunkt von $Q_{k}$ in bezug auf eine Kugel vom Mittelpunkt $P_{0}$, so ist $P_{0}$ der Schwerpunkt der Punkte $Q_{k}^{\prime}(k=1,2, \cdots, n)$. Die Vektoren $P_{0} Q_{k}^{\prime}$ lassen sich in ein Raumvieleck zusammensetzen, weil ihre Summe verschwindet.

4. Die Lage der Nullstellen der Derivierten eines Abstandspolynoms $F(x, y, z)$ in der Umgebung einer Nullstelle von $F(x, y, z)$. Bedeutet $|P Q|$ den Abstand der Punkte $P, Q$, so kann man aus dem Satz III den Satz ableiten:

IV. Bezeichnen $Q_{1}, Q_{2}, \cdots, Q_{m}$ bzw. $p_{1}, p_{2}, \cdots, p_{m}$ die verschiedenen Nullstellen des Abstandspolynoms $F(x, y, z)$ n-ten Grades bzw. ihre Vielfachheiten $\left(p_{k} \geqq 1, p_{1}+p_{2}+\cdots+p_{m}=n\right)$ und bezeichnet $K$ den Konvexkörper der gemeinsamen Punkte $P$ der $m-1$ Kugeln

$$
\left|P Q_{1}\right| \leqq \frac{p_{1}}{n-p_{1}}\left|P Q_{k}\right| \quad(k=2,3, \cdots, m),
$$

so besitzt die Derivierte $F^{\prime}(x, y, z)$ im Innern des Körpers $K$ ausserhalb von $Q_{1}$ keine Nullstelle.

Ist $d=\operatorname{Min}\left|Q_{1} Q_{k}\right|(k=2,3, \cdots, m)$, so ist die Kugel vom Mittelpunkt $Q_{1}$ und vom Halbmesser $p_{1} \cdot d / n$ ein Teilkörper von $K$. Sie enthält ausserhalb von $Q_{1}$ keine Nullstelle der Derivierten $F^{\prime}(x, y, z)$ im Innern.

Für einen inneren Punkt $P_{0}\left(\neq Q_{1}\right)$ des Körpers $K$ kann nämlich die Vektorgleichung

$$
P_{0} N=\sum_{k=1}^{m} p_{k} \cdot P_{0} Q_{k}^{\prime}=p_{1} \cdot P_{0} Q_{1}^{\prime}+\sum_{k=2}^{m} p_{k} \cdot P_{0} Q_{k}^{\prime}=0
$$

nicht bestehen, weil

$$
\left|P_{0} N\right| \geqq p_{1} \cdot\left|P_{0} Q_{1}^{\prime}\right|-\sum_{k=2}^{m} p_{k} \cdot\left|P_{0} Q_{k}^{\prime}\right|>0
$$

ist.

Aus den Annahmen des Satzes IV folgen nämlich die Ungleichungen

$$
\frac{\left|P_{0} Q_{k}^{\prime}\right|}{\left|P_{0} Q_{1}^{\prime}\right|}=\frac{\left|P_{0} Q_{1}\right|}{\left|P_{0} Q_{k}\right|}<\frac{p_{1}}{n-p_{1}} \quad(k=2,3, \cdots, m),
$$




$$
\begin{gathered}
\sum_{k=2}^{m} p_{k}\left|P_{0} Q_{k}^{\prime}\right|<\frac{p_{1}}{n-p_{1}}\left(p_{2}+p_{3}+\cdots+p_{m}\right) \cdot\left|P_{0} Q_{1}^{\prime}\right|=p_{1}\left|P_{0} Q_{1}^{\prime}\right|, \\
\left|P_{0} N\right| \geqq p_{1}\left|P_{0} Q_{1}^{\prime}\right|-\sum_{k=2}^{m} p_{k}\left|P_{0} Q_{k}^{\prime}\right|>0 .
\end{gathered}
$$

Damit ist der Satz IV bewiesen.

5. Die Nullstellenverteilung eines Abstandspolynoms $F(x, y, z)$ in zwei Kugeln, die sich in einer Nullstelle der Derivierten von aussen berühren. Es gilt die folgende Verallgemeinerung eines Satzes ${ }^{1}$ von mir über die Polynome mit einer komplexen Veränderlichen:

V. Bezeichnet $P_{0}=\left(x_{0}, y_{0}, z_{0}\right)$ eine Nullstelle der Derivierten eines Abstandspolynoms $F(x, y, z)$ n-ten Grades (für welche $F\left(x_{0}, y_{0}, z_{0}\right) \neq 0$ ist), bezeichnen ferner $K$ bzw. $K^{\prime}$ die abgeschlossenen Inneren von zwei sich im Punkte $P_{0}$ von aussen berïhrenden beliebigen Kugeln mit dem Halbmesser $R$ bzw. $R^{\prime}=(n-1) R$ und besitzt endlich das Abstandspolynom $F(x, y, z)$ in $K$ mindestens eine Nullstelle, so besitzt es auch in $K^{\prime}$ mindestens eine. Enthält $K^{\prime}$ keine Nullstelle von $F(x, y, z)$, so enthält auch $K$ keine.

Zum Beweis dieses Satzes kann man annehmen, das $x_{0}=y_{0}=z_{0}=0$ sind und dass die Berührungsebene der Kugeln $K$ und $K^{\prime}$ im Punkte $P_{0}$ die $y z$-Ebene ist, weil man diese Lage durch eine Koordinatentransformation erreichen kann. Bei dieser Annahme hat die erste Gleichung von (12) die Form

$$
\sum_{k=1}^{n} \frac{x_{k}}{x_{k}^{2}+y_{k}^{2}+z}=\sum_{k=1}^{n} X_{k}=0, \quad X_{k}=\frac{x_{k}}{x_{k}^{2}+y_{k}+z_{k}^{2}} .
$$

Ist $X_{k}=0$, so liegt die Nullstelle $Q_{k}$ in der $y z$-Ebene. Liegt ein Punkt $Q_{k}$ ausserhalb der $y z$-Ebene, so besitzt die Summe von (14) mindestens je ein positives und negatives Glied.

Wir nehmen an, dass die Indizes der Punkte $Q_{k}$ so gewählt sind, dass die ersten $\nu$ Glieder in der Summe von (14) positiv, die folgenden $\nu^{\prime}$ Glieder negativ und die letzten $n-\nu-\nu^{\prime}$ Glieder Null sind $(\nu \geqq 1$, $\nu^{\prime} \geqq 1, n-\nu-\nu^{\prime} \geqq 0$ ) und dass die Ungleichungen

$$
\begin{gathered}
X_{1} \geqq X_{2} \geqq \cdots \geqq X_{\nu}>0, \quad X_{1}^{\prime} \geqq X_{2}^{\prime} \geqq \cdots \geqq X_{\nu}^{\prime}>0, \\
X_{i}^{\prime}=-X_{\nu+i} \quad\left(i=1,2, \cdots, \nu^{\prime}\right)
\end{gathered}
$$

bestehen. Dann liegen die Punkte $Q_{1}, Q_{2}, \cdots, Q_{\nu}$ bzw. $Q_{\nu+1}$,

${ }^{1}$ Gy. (F.) v. Sz. Nagy, Über geometrische Relationen zwischen den Wurzeln einer algebraischen Gleichung und ihrer Derivierten, Jber. Deutschen Math. Verein. bd. 27 (1918) pp. 44-88; Über die Lage der Nullstellen der Derivierten eines Polynoms, Tôhoku Math. J. bd. 35 (1932) pp. 126-135. 
$Q_{\nu+2}, \cdots, Q_{v+v^{\prime}}$ an der positiven bzw. negativen Seite der $y z$-Ebene, die übrigen Punkte $Q_{v+\nu^{\prime}+1}, \cdots, Q_{n}$ liegen in der $y z$-Ebene.

Sind

$$
X_{h}=1 / D_{h}, \quad X_{i}^{\prime}=1 / D_{i}^{\prime} \quad\left(h=1,2, \cdots, \nu ; i=1,2, \cdots, \nu^{\prime}\right)
$$

so lässt sich die Gleichung (14) in der Form

$$
\begin{gathered}
\sum_{h=1}^{\nu} \frac{1}{D_{h}}=\sum_{i=1}^{\nu} \frac{1}{D_{i}^{\prime}}, \quad \frac{1}{D_{1}} \geqq \frac{1}{D_{2}} \geqq \cdots \geqq \frac{1}{D_{\nu}}, \\
\frac{1}{D_{1}^{\prime}} \geqq \frac{1}{D_{2}^{\prime}} \geqq \cdots \geqq \frac{1}{D_{v^{\prime}}}
\end{gathered}
$$

schreiben. Hier bedeutet $D_{h}$ bzw. $D_{i}^{\prime}$ den Durchmesser der durch den Punkt $Q_{h}$ bzw. $Q_{v+i}$ gehenden und die $y z$-Ebene im Anfangspunkt berührenden Kugel.

Man erhält aus (15) die Ungleichungen

$$
\frac{1}{D_{1}} \leqq \frac{\nu^{\prime}}{D_{1}^{\prime}} \leqq \frac{n-1}{D_{1}^{\prime}}, \quad \frac{p}{D_{p}} \leqq \frac{\nu^{\prime}}{D_{1}^{\prime}} \leqq \frac{n-1}{D_{1}^{\prime}} \quad(p \leqq \nu),
$$

oder

$$
D_{1}^{\prime} \leqq \nu^{\prime} \cdot D_{1} \leqq(n-1) \cdot D_{1}, \quad D_{1}^{\prime} \leqq \frac{\nu^{\prime}}{p} D_{p} \leqq \frac{n-p}{p} D_{p} .
$$

Aus der Ungleichung $D_{1}^{\prime} \leqq(n-1) D_{1}$ folgt der Satz V. In dieser Ungleichung besteht das Gleichheitszeichen nur dann, wenn $\nu=1$, $\nu^{\prime}=n-1, \quad D_{1}^{\prime}=D_{2}^{\prime}=\cdots=D_{\nu}^{\prime}$ sind. Liegt der Punkt $Q_{1}$ auf der Kugel $K\left(D_{1}=2 R\right)$ und liegt kein Punkt $Q_{k}$ innerhalb der Kugel $K^{\prime}\left(D_{1}^{\prime}=2 R^{\prime}\right)$, so fallen die $n-1$ Punkte $Q_{2}, Q_{3}, \cdots, Q_{n}$ auf die Kugel $K^{\prime}$.

Aus der zweiten Ungleichung von (16) folgt die folgende Verallgemeinerung des Satzes V:

VI. Bezeichnet $P_{0}=\left(x_{0}, y_{0}, z_{0}\right)$ eine Nullstelle der Derivierten eines Abstandspolynoms $F(x, y, z)$ n-ten Grades, für welche $F\left(x_{0}, y_{0}, z_{0}\right) \neq 0$ ist, bezeichnen ferner $K$ und $K^{\prime}$ sich im Punkte $P_{0}$ von aussen berïhrende Kugeln von Halbmessern $R$ bzw. $R^{\prime}=(n-p) / p \cdot R$ und besitzt das Abstandspolynom $F(x, y, z)$ in der abgeschlossenen Kugel $K$ mindestens $p$ Nullstellen, so besitzt es in der Kugel $K^{\prime}$ mindestens eine Nullstelle. Besitzt das Abstandspolynom $F(x, y, z)$ im Innern von $K^{\prime}$ keine Nullstelle, so besitzt es auf der Kugel $K$ bzw. $K^{\prime}$ genau $p$ bzw. $n-p$ Nullstellen.

Ist $\nu=\nu^{\prime}=1$, so ist $D_{1}=D_{1}^{\prime}$. Es gilt dann der Satz V: 
VII. Besitzt ein Abstandspolynom n-ten Grades die Nullstellen $Q_{1}$ und $Q_{2}$ ausserhalb einer Ebene $E$, die übrigen $n-2$ Nullstellen $Q_{3}, Q_{4}, \cdots, Q_{n}$ aber in der Ebene $E$ und enthält die Ebene $E$ eine Nullstelle $P_{0}$ der Derivierten $\left(P_{0} \neq Q_{k}, k=1,2, \cdots, n\right)$, so haben die Kugeln, die durch den Punkt $Q_{1}$ bzw. $Q_{2}$ gehen und im Punkte $P_{0}$ die Ebene E berühren, gleiche Durchmesser.

Aus der Gleichung (15) erhält man leicht den Satz

VIII. Es sei $P_{0}$ eine Nullstelle der Derivierten eines Abstandspolynoms 4-ten Grades, deren Nullstellen $Q_{1}, Q_{2}, Q_{3}, Q_{4}$ nicht in einer Ebene liegen. Ist $K_{234}$ die Umkugel des Tetraeders $P_{0} Q_{2} Q_{3} Q_{4}$ und ist $K_{1}$ die Kugel durch den Punkt $Q_{1}$, von der die Kugel $K_{234}$ im Punkte $P_{0}$ beriihrt wird, so ist $R_{234}=3 \cdot R_{1}$, wo $R_{234}$ bzw. $R_{1}$ den Halbmesser von $K_{234}$ bzw. $K_{1}$ bedeutet. Bezeichnet $E_{34}$ die Ebene der Punkte $P_{0} Q_{3} Q_{4}$ und bezeichnet $K_{1}^{\prime \prime}$ bzw. $K_{2}^{\prime}$ die Kugel durch den Punkt $Q_{1}$ bzw. $Q_{2}$, von der die Ebene $E_{34}$ im Punkte $P_{0}$ berïhrt wird, so ist $R_{1}^{\prime \prime}=R_{2}^{\prime}$ (wo $R_{1}^{\prime \prime}$ und $R_{2}^{\prime}$ die Halbmesser von $K_{1}^{\prime \prime}$ und $K_{2}^{\prime}$ bedeuten). Bezeichnet $E_{12,34}$ die durch den $P$ unkt $P_{0}$ gehende und zu den Geraden $Q_{1} Q_{2}$ und $Q_{3} Q_{4}$ parallele Ebene und bezeichnet $K_{12}$ bzw. $K_{34}$ die Kugel durch das Punktpaar $Q_{1} Q_{2}$ bzw. $Q_{3} Q_{4}$, von der die Ebene $E_{12,34}$ im Punkte $P_{0}$ beriuhrt wird, so ist $R_{12}=R_{34}$ (wo $R_{12}$ und $R_{34}$ die Halbmesser von $K_{12}$ und $K_{34}$ sind).

Im ersten, zweiten bzw. dritten Teil dieses Satzes sind nämlich

$\nu=3, \quad \nu^{\prime}=1, \quad D_{1}=D_{2}=D_{3}=2 R, \quad D_{1}^{\prime}=2 R^{\prime}, \quad D_{1}=3 D_{1}^{\prime} ;$

$\nu=\nu^{\prime}=1, \quad D_{1}=D_{1}^{\prime}, \quad D_{1}=2 R, \quad D_{1}^{\prime}=2 R ;$

bzw.

$\nu=\nu^{\prime}=2, \quad D_{1}=D_{2}, \quad D_{1}^{\prime}=D_{2}^{\prime}, \quad D_{1}=D_{1}^{\prime}, \quad D_{1}=2 R, \quad D_{1}^{\prime}=2 R^{\prime}$.

Damit ist der Satz VIII bewiesen.

6. Der Satz von Laguerre für ein Abstandspolynom. Es gilt der Satz:

IX. Ist $P=(x, y, z)$ keine Nullstelle des Abstandspolynoms $F(x, y, z)$ oder seiner Derivierten, so werden die Nullstellen von $F(x, y, z)$ von jeder Kugelfläche geirennt, die den Punkt $P$ und den Punkt $\Pi=(\xi, \eta, \zeta)$

$$
\begin{gathered}
\xi=x-2 n \frac{F_{x}(x, y, z)}{H(x, y, z)}, \quad \eta=y-2 n \frac{F_{y}(x, y, z)}{H(x, y, z)}, \\
\zeta=z-2 n \frac{F_{z}(x, y, z)}{H(x, y, z)}
\end{gathered}
$$


enthält, wo $H(x, y, z)=F_{x}^{2}(x, y, z)+F_{y}^{2}(x, y, z)+F_{z}^{2}(x, y, z)$ ist.

Der Punkt $\Pi=(\xi, \eta, \zeta)$ wird als Polarpunkt von $P$ in bezug auf das Abstandspolynom $F(x, y, z)$ genannt. Der Punkt $\Pi$ kann nach Laguerre als der derivierte Punkt ${ }^{2}$ von $P$ in bezug auf $F(x, y, z)$, oder nach Pólya-Szegö als der Schwerpunkt ${ }^{3}$ der Nullstellen von $F(x, y, z)$ in bezug auf $P$ genannt werden.

In diesem Satz sind die Ebenen durch das Punktpaar $P I I$ den Kugeln zu rechnen. Die Kugelfläche $K$ trennt dann die Nullstellen von $F(x, y, z)$, wenn sie jede dieser Nullstellen enthält, oder wenn das Abstandspolynom $F(x, y, z)$ innerhalb und ausserhalb von $K$ mindestens je eine Nullstelle besitzt.

Zum Beweis des Satzes IX können wir annehmen, dass $P=(0,0,0)$ ist. Haben die Nullstellen $Q_{k}$ von $F(x, y, z)$ in diesem Koordinatensystem die Koordinaten $\left(x_{k}, y_{k}, z_{k}\right)(k=1,2, \cdots, n)$, so hat der Spiegelpunkt $Q_{k}^{\prime}$ von $Q_{k}$ in bezug auf die Einheitskugel (vom Mittelpunkt $P=(0,0,0)$ und vom Einheitshalbmesser) die Koordinaten

$$
x_{k}^{\prime}=\frac{x_{k}}{x_{k}^{2}+y_{k}^{2}+z_{k}^{2}}, \quad y_{k}^{\prime}=\frac{y_{k}}{x_{k}^{2}+y_{k}^{2}+z_{k}^{2}}, \quad z_{k}^{\prime}=\frac{z_{k}}{x_{k}^{2}+y_{k}^{2}+z_{k}^{2}} .
$$

Der Schwerpunkt $S^{\prime}=\left(X^{\prime}, Y^{\prime}, Z^{\prime}\right)$ der $n$ Punkte $Q_{k}^{\prime} \quad(k=1$, $2, \cdots, n$ ) hat nach $(10)$ die Koordinaten

$$
\begin{gathered}
X^{\prime}=\frac{1}{n} \sum_{k=1}^{n} x_{k}^{\prime}=\frac{-1}{2 n} \cdot \frac{F_{x}(0,0,0)}{F(0,0,0)}, \quad Y^{\prime}=\frac{-1}{2 n} \cdot \frac{F_{y}(0,0,0)}{F(0,0,0)}, \\
Z^{\prime}=\frac{-1}{2 n} \cdot \frac{F_{z}(0,0,0)}{F(0,0,0)} .
\end{gathered}
$$

Ist $S=(X, Y, Z)$ der Spielgelpunkt von $S^{\prime}=\left(X^{\prime}, Y^{\prime}, Z^{\prime}\right)$ in bezug auf die Einheitskugel, so fällt $S$ mit dem Punkt II zusammen, weil

$$
\begin{gathered}
X=\frac{X^{\prime}}{X^{\prime 2}+Y^{\prime 2}+Z^{\prime 2}}=\frac{-2 n \cdot F_{x}(0,0,0)}{H(0,0,0)}, \quad Y=\frac{-2 n \cdot F_{y}(0,0,0)}{H(0,0,0)}, \\
Z=\frac{-2 n \cdot F_{z}(0,0,0)}{H(0,0,0)}
\end{gathered}
$$

sind.

Das Punktsystem $Q_{1}^{\prime}, Q_{2}^{\prime}, \cdots, Q_{n}^{\prime}$ wird von jeder durch seinen Schwerpunkt $S^{\prime}$ gehenden Ebene $E$ getrennt. Die Spiegelung in bezug auf die Einheitskugel führt die Ebene $E$ in eine die Punkte $P$ und $S \equiv \Pi$ enthaltende Kugelfä̈che $K$, die Punkte $Q_{1}^{\prime}, Q_{2}^{\prime}, \cdots, Q_{n}^{\prime}$

${ }^{2}$ E. Laguerre, Oeuvres de Laguerre, bd. II, pp. 56-63.

${ }^{3}$ G. Pólya und G. Szegö, Aufgaben und Lehrsätze aus der Analysis, bd. II, pp. 55-66. 
in die Punkte $Q_{1}, Q_{2}, \cdots, Q_{n}$ über, die also von der Kugelfläche $K$ getrennt werden. Damit ist der Satz IX bewiesen.

7. Abstandspolynom mit in bezug auf eine Ebene symmetrisch liegenden Nullstellen. Der bekannte Jensensche Satz ${ }^{4}$ lässt sich auf folgende Weise verallgemeinern:

X. Ist das Nullstellenpaar $Q_{2 h-1} Q_{2 h}(h=1,2, \cdots, p)$ eines $A b$ standspolynoms $F(x, y, z)$ n-ten Grades symmetrisch in bezug auf eine Ebene $E$ und liegen seine ïbrigen Nullstellen $Q_{k}(k=2 p+1$, $2 p+2, \cdots, n)$ in der Ebene $E$, so fällt eine ausserhalb der Ebene $E$ liegende und von den Punkten $Q_{i}(i=1,2, \cdots, 2 p)$ verschiedene Nullstelle $P$ der Derivierten $F^{\prime}(x, y, z)$ entweder in das Innere von mindestens einer der $p$ Kugeln mit den Durchmessern $Q_{2 h-1} Q_{2 h}$ $(h=1,2, \cdots, p)$, oder $P$ ist ein gemeinsamer Punkt dieser $p$ Kugelflächen.

Zum Beweis dieses Satzes können wir annehmen, dass die Ebene $E$ mit der $x y$-Ebene zusammenfält, dass also

$$
\begin{array}{rlr}
Q_{2 h-1} & =\left(x_{h}, y_{h}, z_{h}\right), \quad Q_{2 h}=\left(x_{h}, y_{h},-z_{h}\right) \quad\left(z_{h} \neq 0, h=1,2, \cdots, p\right) ; \\
Q_{k} & =\left(x_{k}, y_{k}, 0\right) & (k=2 p+1,2 p+2, \cdots, n)
\end{array}
$$

sind. Ist $P=(x, y, z)$ im Satz $\mathrm{X}$, so sind $F^{\prime}(x, y, z)=0, F(x, y, z) \neq 0$ und $z \neq 0$. Aus (10) ergibt sich die Gleichung

$$
\begin{aligned}
\frac{F_{z}(x, y, z)}{2 F(x, y, z)}= & \sum_{h=1}^{p}\left[\frac{z-z_{h}}{\left(x-x_{h}\right)^{2}+\left(y-y_{h}\right)^{2}+\left(z-z_{h}\right)^{2}}\right. \\
& \left.+\frac{z+z_{h}}{\left(x-x_{h}\right)^{2}+\left(y-y_{h}\right)^{2}+\left(z+z_{h}\right)^{2}}\right] \\
& +\sum_{k=2 p+1}^{n} \frac{z}{\left(x-x_{k}\right)^{2}+\left(y-y_{k}\right)^{2}+z^{2}} \\
= & 2 z\left\{\sum_{h=1}^{p} \frac{\left(x-x_{h}\right)^{2}+\left(y-y_{h}\right)^{2}+z^{2}-z_{h}^{2}}{\left[\left(x-x_{h}\right)^{2}+\left(y-y_{h}\right)^{2}+\left(z-z_{h}\right)^{2}\right]}\right. \\
& \left.+\frac{1}{2} \sum_{k=2 p+1}^{n} \frac{1}{\left(x-x_{k}\right)^{2}+\left(y-y_{k}\right)^{2}+z^{2}}\right\}=0 .
\end{aligned}
$$

${ }^{4}$ L. W. Jensen, Recherches sur la théorie des équations, Acta Math. bd. 36 (1913) pp. 181-195.

Gy. (F.) v. Sz. Nagy, Zur Theorie der algebraischen Gleichungen, Jber. Deutschen Math. Verein. bd. 31 (1922) pp. 258-251. 
Der Faktor von $2 z(z \neq 0)$ kann nur dann verschwinden, wenn die erste Summe mindestens ein Glied mit einem negativen Zähler besitzt, oder wenn jedes Glied der erste Summe verschwindet und die zweite Summe kein Glied enthält $(n=2 p)$.

Damit ist der Satz X bewiesen.

Der Faktor $2 z$ verändert sich nicht, wenn man den Punkt $P=(x, y, z)$ durch den Punkt $P^{\prime}=(x, y,-z)$ ersetzt. Ebenso bleiben die Summen in den ersten zwei Gleichungen von (10) bei dieser Ersetzung unverändert. Daraus folgt, dass auch die Lage der Nullstellen der Derivierten $F^{\prime}(x, y, z)$ in bezug auf die Ebene $E$ symmetrisch ist.

Aus dem Satz $\mathrm{X}$ folgt:

XI. Liegen die Nullstellen $Q_{1}, Q_{2}, \cdots, Q_{n}$ eines Abstandspolynoms n-ten Grades in einer Ebene $E$, sind die Punktpaare $Q_{2 h-1} Q_{2 h}$ $(h=1,2, \cdots, p)$ in bezug auf eine Gerade $g$ der Ebene E symmetrisch und fallen die übrigen $n-2 p$ Punkte $Q_{k}$ auf die Gerade $g$, so ist jede ausserhalb von $g$ liegende und von den Punkten $Q_{k}(k \leqq 2 p)$ verschiedene Nullstelle der Derivierten ein Punkt von mindestens einer Kreisscheibe unter den $p$ Kreisscheiben mit den Durchmessern $Q_{2 h-1} Q_{2 h}$.

Die Nullstellen des Abstandspolynoms in diesem Satz sind nämlich auch in bezug auf die Ebene $E^{\prime}$ symmetrisch, die durch die Gerade $g$ geht und zu $E$ senkrecht steht.

8. Die Lage der Nullstellen eines Abstandspolynoms $F(x, y, z)$ in bezug auf zwei Nullstellen seiner Derivierten. Es gilt der Satz:

XII. Bezeichnen $P_{0}=\left(x_{0}, y_{0}, z_{0}\right)$ und $P_{0}^{\prime}=\left(x_{0}^{\prime}, y_{0}^{\prime}, z_{0}^{\prime}\right)$ zwei verschiedene Nullstellen der Derivierten eines Abstandspolynoms $F(x, y, z)$ n-ten Grades, für welche $F\left(x_{0}, y_{0}, z_{0}\right)$ und $F\left(x_{0}^{\prime}, y_{0}^{\prime}, z_{0}^{\prime}\right)$ nicht verschwinden und bezeichnet $E$ bzw. $E_{0}$ eine beliebige Ebene durch die Punkte $P_{0}$ und $P_{0}^{\prime}$, bzw. die Mittelebene der Punkte $P_{0}, P_{0}^{\prime}$, so trennt das Ebenenpaar $E E_{0}$ die Nullstellen von $F(x, y, z)$. (Enthält das Ebenenpaar nicht jede Nullstelle von $F(x, y, z)$, so besitzt $F(x, y, z)$ im Inneren beider von $E$ und $E_{0}$ begrenzten Doppel-Raumwinkel mindestens je eine Nullstelle.)

Dreht man eine gleichseitige Hyperbel mit der Hauptachse $P_{0} P_{0}^{\prime} u m$ die Gerade $P_{0} P_{0}^{\prime}$ herum, so trennt das so enstandene zweischalige Hyperboloid die Nullstellen des Abstandspolynoms $F(x, y, z)$.

Zum Beweis dieses Satzes können wir annehmen, dass $P_{0}=\left(0,0, z_{0}\right)$, $P_{0}^{\prime}=\left(0,0,-z_{0}\right), z_{0} \neq 0$ sind. Bezeichnen $Q_{k}=\left(x_{k}, y_{k}, z_{k}\right)$ die Nullstellen von $F(x, y, z)$ in diesem Koordinatensystem und ist 


$$
\begin{aligned}
N_{k}=\left[x_{k}^{2}+y_{k}^{2}+\left(z_{k}-z_{0}\right)^{2}\right] \cdot\left[x_{k}^{2}+y_{k}^{2}+\left(z_{k}+z_{0}\right)^{2}\right] & \\
& (k=1,2, \cdots, n),
\end{aligned}
$$

so erhält man aus (10) die Gleichungen

$$
\begin{aligned}
G_{1} & \equiv \frac{1}{2 z_{0}}\left[\frac{F_{x}\left(0,0, z_{0}\right)}{F\left(0,0, z_{0}\right)}-\frac{F_{x}\left(0,0,-z_{0}\right)}{F\left(0,0,-z_{0}\right)}\right] \\
& =\sum_{k=1}^{n} \frac{-2 x_{k} z_{k}}{N_{k}}=0, \\
G_{2} & \equiv \frac{1}{2 z_{0}}\left[\frac{F_{y}\left(0,0, z_{0}\right)}{F\left(0,0, z_{0}\right)}-\frac{F_{y}\left(0,0,-z_{0}\right)}{F\left(0,0,-z_{0}\right)}\right] \\
& =\sum_{k=1}^{n} \frac{-2 y_{k} z_{k}}{N_{k}}=0, \\
G_{3} & \equiv \frac{1}{2 z_{0}}\left[\frac{F_{z}\left(0,0, z_{0}\right)}{F\left(0,0, z_{0}\right)}-\frac{F_{z}\left(0,0,-z_{0}\right)}{F\left(0,0,-z_{0}\right)}\right] \\
& =\sum_{k=1}^{n} \frac{x_{k}^{2}+y_{k}^{2}-z_{k}^{2}+z_{0}^{2}}{N_{k}}=0 .
\end{aligned}
$$

Bezeichnen $A$ und $B$ beliebige reele Zahlen, so ist

$$
A G_{1}+B G_{2} \equiv-\sum_{k=1}^{n} \frac{\left(A x_{k}+B y_{k}\right) z_{k}}{N_{k}}=0 \text {. }
$$

Daraus folgt der erste Teil des Satzes XII. Verschwindet nämlich das Produkt $(A x+B y) z$ nicht für jede Nullstelle $Q_{k}$, so gibt es mindestens je einen Punkt $Q_{h}$ bzw. $Q_{i}$, für den dieses Produkt positiv bzw. negativ ist. Das Punktpaar $Q_{h} Q_{i}$ wird also von den Ebenen $A x+B y$ $=0$ und $z=0$ getrennt. Ebenso folgt der zweite Teil von XII aus der Gleichung $G_{3}=0$.

Aus der Gleichung $A \cdot G_{1}+B \cdot G_{2}+G_{3}=0$ folgt die folgende Ergänzung des Satzes XII:

Die Nullstellen des Abstandspolynoms $F(x, y, z)$ werden im Falle $P_{0}=\left(0,0, z_{0}\right), P_{0}^{\prime}=\left(0,0,-z_{0}\right)$ von jeder Fläche zweiter Ordnung mit der Gleichung

$$
(x-A z)^{2}+(y-B z)^{2}-\left(1+A^{2}+B^{2}\right) z^{2}+z_{0}^{2}=0
$$

getrennt, wo $A$ und $B$ beliebige reele Zahlen bedeuten.

Diese Flächen sind im allgemeinen zweischalige Hyperboloide. Im Grenzfall $A \rightarrow \infty, B \rightarrow \infty$, stellt die Gleichung (18) zwei senkrechte Ebenen dar. 
Dieser Satz ist eine Verallgemeinerung eines Satzes von mir. ${ }^{5}$

9. Abstandspolynome in den mehrdimensionalen euklidischen Räumen. Ein Abstandspolynom $n$-ten Grades in dem $m$-dimensionalen euklidischen Raum ist eine reelle Funktion der $m$ reellen Veränderlichen $x_{1}, x_{2}, \cdots, x_{m}$ von der Form

$$
\begin{array}{r}
F\left(x_{1}, x_{2}, \cdots, x_{m}\right) \\
=C \prod_{k=1}^{n}\left[\left(x_{1}-x_{1, k}\right)^{2}+\left(x_{2}-x_{2, k}\right)^{2}+\cdots+\left(x_{m}-x_{m, k}\right)^{2}\right], \\
C>0 .
\end{array}
$$

Die Punkte $Q_{k}=\left(x_{1, k}, x_{2, k}, \cdots, x_{m, k}\right) \quad(k=1,2, \cdots, n)$ sind die Nullstellen des Abstandspolynoms.

Die Derivierte des Abstandspolynom (19) wird durch die Gleichung

$$
F^{\prime}\left(x_{1}, x_{2}, \cdots, x_{m}\right)=\frac{1}{4 \cdot F} \sum_{h=1}^{m} F_{x_{h}}^{2}, \quad F_{x_{h}}=\frac{\partial F}{\partial x_{h}}
$$

definiert. Man sieht ebenso ein, wie im Falle $m=3$, dass $F^{\prime}$ ein Polynom 2(n-1)-ten Grades der $m$ Veränderlichen $x_{1}, x_{2}, \cdots, x_{m}$ ist.

Unsere Sätze lassen sich ohne grössere Schwierigkeiten auch für $m>3$ verallgemeinern.

UNIVERSITY OF SZEgEd, Hungary

5 Vgl. meine in der Fussnote 4 angeführte Arbeit. 